\title{
PROVISIONAL RESTORATIONS IN RESTORATIVE DENTISTRY
}

\author{
Artak G. Heboyan, Assistant Professor, Department of Prosthodontics, Yerevan State Medical \\ University after M. Heratsi; Yerevan, Armenia; \\ Narek M. Movsisyan, Prosthodontist, Private Practice, Yerevan, Armenia; \\ Victor A. Khachatryan, Prosthodontist, Private Practice, Yerevan, Armenia
}

DOI: https://doi.org/10.31435/rsglobal_ws/30062019/6570

\begin{abstract}
ARTICLE INFO
Received: 13 April 2019

Accepted: 11 June 2019

Published: 30 June 2019

\section{KEYWORDS}

provisional restorations, temporary restorations, interim prosthesis, interim treatment.

ABSTRACT

Provisional restorations are of a great significance in fixed prosthodontic rehabilitation, particularly in case long-term treatment is needed before final prosthetics. The restorations are also important from the point of view of diagnosis and treatment plan assessment so they should be similar to the final constructions in their shape and function. Provisional restorations quite often need alterations and adjustment to the new requirements, so it's important to comprehend the compositional properties of the base and repair materials to make a reliable bond between them. Thus, interim treatment should meet a number of requirements such as biocompatibility, marginal adaptation, strength and longevity. The purpose of this review is to discuss fabrication technology of provisional restorations, possible failure and complications, the ways to correct them as well as the aspects of relining provisional restoration.
\end{abstract}

Citation: Artak G. Heboyan, Narek M. Movsisyan, Victor A. Khachatryan. (2019) Provisional Restorations in Restorative Dentistry. World Science. 6(46), Vol.3. doi: 10.31435/rsglobal_ws/30062019/6570

Copyright: (C) 2019 Artak G. Heboyan, Narek M. Movsisyan, Victor A. Khachatryan. This is an openaccess article distributed under the terms of the Creative Commons Attribution License (CC BY). The use, distribution or reproduction in other forums is permitted, provided the original author(s) or licensor are credited and that the original publication in this journal is cited, in accordance with accepted academic practice. No use, distribution or reproduction is permitted which does not comply with these terms.

Introduction. According to the Glossary of Prosthodontic Terms, "provisional or interim prosthesis or restoration is a fixed or removable dental or maxillofacial prosthesis designed to enhance esthetics, stabilization and/or function for a limited period of time, after which it is to be replaced by a definitive dental or maxillofacial prosthesis" [1]. Fabrication of provisional restorations plays an important role in fixed prosthodontics. Interim treatment includes fabrication of provisional restorations which are particularly important in case of full mouth reconstruction, where a number of teeth are prepared. In such cases provisional restorations frequently remain in the oral cavity for comparatively long (6-12 weeks), which is necessary in order to assess the patient's contentment and comfort as well as to make necessary adjustment $[2,3]$. The goal of interim treatment is to protect the pulp and periodontium, to promote guided tissue healing in to achieve an acceptable emergence profile, to evaluate hygiene procedures, to prevent displacement of abutments, to improve esthetics and correct pronunciation, provide adequate occlusal scheme and evaluate intermaxillary relationship [4]. The choice of material for provisional restoration should depend on how their mechanical, physical and handling properties fulfill specific requirements of any clinical case. Biocompatibility and complications from intraoral use, such as chemical injury from the presence of monomer residue and thermal injury from an exothermic polymerization reaction should be also taken into consideration. The most common materials used for custom interim-fixed restorations are several types of acrylic resins such as polymethyl methacrylate (PMMA) resin, polyethyl methacrylate (PEMA) resin, polyvinyl methacrylate resin, bis-acryl composite resin, visible light-cured urethane dimethacrylates and microfill resin [5-8]. An adequate marginal adaptation, fracture resistance, low thermal conductivity, a non-irritant reaction with dental pulp and gingival tissue and ease of cleaning are indispensable requirements to provisional materials. In oral cavity, the majority of bacteria can flourish sticking around the teeth, the surfaces of filling materials, dental implants or prosthesis, each 
having various peculiarities [9]. Thus, provisional restorations should satisfy a number of requirements such as proper marginal fit, prevention of the pulp and periodontium injury, abutment stability, dimensional stability, occlusal function recovery, ability to be cleansed, wear resistance, strength, ease of contour and repair as well as esthetic aspects [10].

The objective of the review is to elucidate fabrication techniques of provisional restorations, to point out possible failures in the process of making restoration and complications they can cause as well as to denote the ways how to avoid and correct them.

\section{Main text.}

\section{Provisional restoration techniques}

Different authors suggest various techniques of making provisional restorations which have improved over the time, having positive psychological influence on the patient's perceived self-esteem and quality of life [11]. Fabrication of provisional restorations requires certain period of time, but this stage should not be neglected. In case of in-office $\mathrm{CAD} / \mathrm{CAM}$ fabrication of restorations the operations are performed outside the oral cavity while the patient is waiting in the waiting room. During this period, examination of their own prepared teeth by the patients might provoke negative attitude and mistrust towards the treatment, therefore, at the stage of treatment planning the doctor should use various visual means such as videos or computer simulations of prepared teeth. Provisional restorations can be made directly on the prepared teeth with the use of a matrix or indirectly by making an impression of the prepared teeth [12]. A combination of indirect-direct technique is also possible which has evolved as a sequential application of these that involves fabrication of a preformed shell that is relined intraorally [1316]. Direct technique is the most frequently used one in fabrication of provisional restorations since it is a quick and relatively low-cost procedure. The marginal accuracy of provisional restorations fabricated by direct technique is more precise, than those made by the indirect technique. However, the main goal of provisional restorations is to prepare properly contoured and well-fitting restoration, which will maintain its integrity throughout the reasonable time from tooth preparation to completion of definitive treatment [17].

\subsection{Indirect provisional restorations}

This technique implies fabrication of provisional restorations outside the oral cavity and no issues typical for direct technique are observed in the process. In this technique of restoration fabrication fast-setting plaster can be used. The technique has some advantages over the direct technique. There is no direct contact of free monomer with prepared teeth and the gum, which can result in the injury of the tissues, allergic reaction or sensitization. Besides, the application of this technique excludes the damage of prepared teeth with the heat released by polymerization. The restorations fabricated by indirect technique have excellent marginal fit, favorable mechanical properties and at the same time save both the doctor's and patient's time. However, this technique has its drawbacks either, such as increased chair side time, increased number of intermediate steps, the need of special equipment. It requires certain laboratory facilities and is more expensive due to its laboratory costs.

\subsection{Indirect-direct provisional restorations}

The technique produces a custom made preformed external surface form of the restoration but the internal tissue surface form if formed by the underprepared diagnostic casts. This technique has certain advantages such as reduction of chair time, since the provisional shell is fabricated before the patient's appointment. Enhanced control over restoration contours allows to reduce the time necessary for chair side adjustments. In addition, a smaller amount of acrylic resin will polymerize in contact with the prepared abutments, resulting in decreased heat generation, chemical exposure, and polymerization shrinkage as compared to the direct technique. Another advantage is the fact that contact between resin monomer and soft tissues is reduced and there are less chances of allergic reactions. The drawback of this technique is the potential need of a laboratory phase before tooth preparation and the adjustments that are frequently needed to seat the shell completely on the prepared tooth.

\subsection{Direct provisional restorations}

In this case the patient's prepared teeth and gingival tissue directly provide the tissue surface form eliminating all the intermediate laboratory procedures. This technique can be applied in case assistant training and the laboratory facilities are inadequate to fabricate efficient indirect restoration. The significant advantages of fabricating restorations with this technique are saving time and low cost, while disadvantages are presence of saliva, insufficient visibility and access, tissue trauma from the polymerizing resin and inherently poorer marginal fit.

\section{Failures in fabrication of provisional restorations and further complications}

Chemical composition of the substances used to fabricate provisional restorations should not produce any negative effect on the soft tissues of oral cavity, causing irritation or damage. In case the 
constructions are made by the direct technique, when self-cured resin is used, the heat released during polymerization can damage the pulp. It takes place as a result of increase in the volume of liquid in dentine canals, change of blood flow direction in the pulp, injury of the vessels, coagulation of protoplasm and tissue necrosis. In the process of fabricating provisional restorations with self-cured resin the temperature of the pulp might increase by $0,42-7,21^{\circ} \mathrm{C}$. The increase of the pulp temperature by $5,6^{\circ} \mathrm{C}$ in $15 \%$ of cases results in the pulp necrosis. When temperature rises by $11,2^{\circ} \mathrm{C}$ the pulp necrosis is observed in $60 \%$ of cases. It makes $100 \%$ in case the temperature rises by $16,8^{\circ} \mathrm{C}$.

The disadvantages of acrylic self-cured resin are high risk of the pulp and periodontium injury with free monomer, noticeable polymerization shrinkage and expressed exothermic reaction. Less heat is released while working with polyethyl methacrylate than when using polymethyl methacrylate, but it's still more than while working with bis-acrylic resins. Many properties of bis-acrylic composite resins of chemical, light and dual curing are better than those of self-cured acrylic resins. They are harder, almost odor free, resistant to discoloration and more wear-resistant.

Taking into consideration all above-mentioned, the doctor should choose the substance for making a provisional crown depending on clinical case. Accordingly, while fabricating provisional restorations by direct technique it's preferable to use polyethyl methacrylic or bis-acrylic compositebased resin endowed with low exothermic reaction, while substances with low polymerization shrinkage (bis-acrylic composite based resin) should be used when indirect technique is applied. While making provisional restorations for the long-term application, it is necessary to use substances of higher stability and durability (polymethyl methacrylic or bis-acrylic composite based resins).

Failures in fabrication of provisional restorations causes lesions of the gum and periodontium. Microorganizms sticking on restoration surface and formation of dental plaque on either the tooth or dental materials are the main causes of oral diseases such as denture stomatitis, gingival inflammation and secondary cavities $[18,19]$. Thus, bacteria play a significant role in the early formation of plaque, not only on the natural tooth tissues but on contemporary resin-based restorative dental materials [20]. Therefore, the surface of provisional restorations should be sufficiently soft in order to be comfortable, esthetically pleasing and capable of avoiding both stains and the accumulation of plaque. Appropriate measures are taken to smooth the rough surface of restorations thus preventing the adhesion of bacteria and occurence of dental plaque.

Soft tissues in their turn can also have an impact on provisional restorations. Particularly, inflamed and bleeding gums decrease the accuracy of the impression taken, while large amount of gingival fluid leads to the cement dissolution at the finish line of provisional restoration causing fixation and impermeability disorders and penetration of bacteria as a result.

In order to provide desirable morphology, antagonist or adjacent teeth proper contact it is often necessary to add a substance. Creating precise external contours ensures proximal and occlusal stability and maintains tooth positions while the restorative plan is executed. Moreover, it gives the patient an opportunity to self-evaluate the appearance, particularly from esthetic aspect and provides a blueprint for the definitive restoration. Alteration of external contours of provisional restorations might be essential after the extraction of the tooth or other surgical intervention. Residual ridge of undesirable shape [21] can be transformed by gradually adding substance to create pressure, resulting in a more favorable tissue configuration (site conditioning) [22].

Provisional restorations should have proper marginal adaptation towards the finish line of the prepared tooth in order to protect the pulp from the harmful effect of thermal, biological, and chemical factors, otherwise long-term contact of the dentin with the external environment leads to penetration of bacteria endangering the vitality of the pulp. Subgingival preparation requires additional support for the free gingival margin to provide the appropriate emergence profile. The accuracy of marginal adaptation of provisional restorations can be conditioned by the substance they're made of. Accordingly, the marginal gap ranges from 25 to $157 \mu \mathrm{m}$. It's difficult to achieve proper marginal adaptation with polymethyl methacrylate. Unlike polymethyl methacrylate, polyethyl methacrylate is less hard but easier to handle and it provides proper marginal adaptation. The quality of marginal adoption of provisional restorations depends on the degree of polymerization shrinkage of the substance. Due to glass fillers bis-acrylic substances undergo less polymerization shrinkage and provide better marginal adaptation. The problems arise when self-cured resin is used, conditioned by the expressed shrinkage of the latter. The volume of polymethyl methacrylate polymerization shrinkage makes up $6 \%$, while it comes to $1-1.7 \%$ in composites, conditioning better marginal adaptation in case the latter is used. The longer the span of the prosthesis, the greater the shrinkage and distortion, resulting in less satisfactory adaptation over the abutments. 
Another factor that contributes to the proper quality of marginal adaptation is fabrication technique. Indirect fabrication provides much better marginal fit compared to direct methods where polymethyl (PMMA) or polyethyl (PEMA) methacrylate resins are used. Direct technique of fabrication is usually performed by successive removals and repositioning of the material over the prepared teeth or bench polymerization after initial set to avoid the trauma of the pulp. The time of the removal plays a crucial role in limiting the material distortion [23].

The marginal adaptation mismatch can be caused by provisional restorations being short which can occur either during construction or as a result of excess preparation of restoration margin. Provisional restorations fit can be also influenced by the moisture of the oral cavity, temperature changes and bite forces $[24,25]$. Moreover, at different stages of the treatment recementation require the removal of temporary cement, followed by inevitable trimming of their intaglio surface in a way that progressively deteriorates the initial marginal accuracy. Finally, the fit and marginal integrity of an existing provisional restoration can be deteriorated when an alteration of the taper or marginal configuration of the prepared tooth occurs. Marginal gaps which occurring in such cases can be reduced by relining the restorations. Adding provisional material allows better adaptation of provisional restoration to the surface of prepared tooth. Relining can be performed at the time of fabrication in order to reduce the polymerization shrinkage of resin and to improve initial retention. The smaller the marginal gap, the less the dissolution of temporary luting cements and plaque accumulation.

The fracture of provisional restorations can take place while removing it from the oral cavity, during construction, trimming or functioning [26]. It can occur as a result of a crack spreading from a surface defect, inadequate transverse strength, impact strength or fatigue resistance [27]. Overload during functional or parafunctional activities can lead to a fracture, especially in a connector area of a long-span interim restoration [28] or where voids have occurred during fabrication. Moreover, insufficient preparation of the tooth brings to thin interim restoration, which is more prone to fracture especially in the cervical area [29]. Fractured connectors and missing margins can deteriorate function, the condition of tooth and soft tissues causing discomfort to the patient [30].

The best way of reducing the risk of fracture is the proper choice of materials taking into consideration their behavior in the oral environment when a subject to aging, fatigue, watersorption and wear processes [31]. Thus, it is important to be aware of the flexural strength of various types of resins for provisional restorations, since many of them are fragile [32]. PMMAs are considered to exhibit higher fracture toughness than bisphenol A glycidyl methacrylate (bis-GMA) resins [33,34]. The resins are widely used though being fragile and prone to fracture when used in long-span bridges. As heat-polymerized acrylic resin materials are denser, stronger, and more resistant to fracture than their auto-polymerized and light-polymerized counterparts, they should be considered for use when provisional treatment for prolonged time or additional strength are required.

The durability of provisional dental resins is increased by means of fibers. Compared to metals, reinforcement with fibers provides excellent mechanical, esthetic and cohesive characteristics and has the advantage of being a lighter-weight composite [35]. The fiber quantity and the reinforcement location rather than the length affect the strengthening efficiency [36]. Preimpregnation of the fibers using the polymer-monomer mix for methacrylates and a bonding agent for bis-acryl resins provides optimal adhesion between the fibers and the polymer matrix, upgrading the strengthening effect.

Making additions to correct many of the deficiencies cited above may require the use of the same material or the combination of different materials [37]. Compatibility problems can occur in terms of bonds occurring between provisional base material and the correcting material. Under these conditions, it is important to process the surface to ensure a bond between two substances.

Chemical similarity of the substances is usually important in polymer repair. The application of provisional base and repair resins of the same chemical composition provides stronger bond between them, as compared to the use of dissimilar ones.

Self-cured acrylic resins are easy to handle and they allow to easily reconstruct the shape defects. However, the use of this substance is manifested with unpleasant odor, significant shrinkage, short working time as well as with pronounced exothermic setting reaction. Moreover, residual methacrylate monomer demonstrates cytotoxicity and potential allergic reactions [38,39]. Heat-cured acrylic resins have lower repair strength compared with self-cured PMMA resin maybe due to fewer free carbon double bonds available for reaction with the PMMA repair resin. The surface design seems to be an important issue in the strength of the repair; however, unprepared surface of PMMA doesn't ensure the bond between the repair surfaces and the self-cured resin. The surface of PMMA-h subject to reconstruction should be moistened with methyl methacrylate monomer or acetone [40], which 
solving the surface allows the diffusion of added acrylic resin, providing reliable bond especially in aged restorations at the expense of new polymer chains.

Bis-acryl resin composites became popular over the last decade due to its easy application, minimum shrinkage and low exothermic reaction [41]. Due to their cartridge dispensing system they have better kneading properties thus providing improved marginal fit. Methacrylate resins used to repair bis-acryl provisional restorations do not bond well with it because of the substance incompatibility [42], thus light-cured flowable resin composites can be used to repair bis-acryl resin composite provisional intraoral restorations. The latter have numerous advantages, among them variety of shades and viscosities, easy handling, sufficient working time, absence of unpleasant odor, low polymerization shrinkage and marginal fit accuracy.

Its rather troublesome to make a reliable bond between the new polymerized composite and aged composite, since primary interfacial bonding between layers of composites decreases as the original layer sets, perhaps because the number of unreacted methacrylate groups decreases during polymerization. When polymerization is over, the solubility and permeability of the polymer decrease reducing the occurrence of bonds between the new composite and the aged composite surfaces. The composition of the base material and repair material, the repair liquid and the surface texture have its impact on the bond strength. Moreover, rough surface contributes to mechanical interlocking, while viscosity of light-cured resins can influence repair strength and low viscosity of repair composite material leads to better adaptation. Thus, a combination of air abrasion and a bonding agent seems to be the most effective protocol for composite-to-composite repairs [43-46]. The bond strength between PMMA and flowable composite can be increased by moistening the surface with a bonding agent in order to make chemical bond between two surfaces [47].

Self-cured acrylic materials or light-cured flowable composite resin materials are used to reline provisional restoration. It's impossible to completely reset most restorations to their previous position due to the interference of hydrostatic pressure caused by the additional resin. The solution to the problem is in venting prior to relining. Otherwise, the part of inner layer of provisional restoration can be removed before adding restorative material in order to create sufficient space to reduce hydrostatic pressure and provide complete fitting of restoration. Removal of cement residue and roughening of the external surface of the margins prior to relining are also very important to ensure good adhesion of the new resin to the aged restoration. Potential damage conditioned by monomer residue and heat release resulted from exothermic reaction can be reduced by lightly spraying water over the area during active polymerization, [48] while retraction cord can be used to improve marginal adaptation in intrasulcular area.

For minor modifications application of acrylic resin with a brush allows for quick and easy correction of external contours of restoration in order to correct a marginal gap, the emergence profile or proximal and occlusal contacts of the provisional restorations. Surface preparation is required, especially in aged restorations. Despite its effectiveness, this technique is time-consuming and has limited working time which makes the modification of multicomponent restorations more complicated [49].

To improve the condition of underlying tissues new layer of acrylic or composite resin is added which works as a valuable management strategy to enhance the tissue conditioning. The amount of resin to be added is determined through an analysis of the shape of the tissue and esthetics.

All temporary cements should provide reliable fixation for provisional restorations, at the same time allowing its easy removal if needed. Previously, zinc oxide-eugenol cements were most often used. Eugenol has certain antibacterial properties and reduces sensitivity of prepared teeth which is preferable in the treatment of vital teeth. However, in case repeated relining is needed after fixation eugenol might interfere with the polymerization of acrylic resin. In case final restoration is planned to be fixed with resin cement eugenol-containing cements are for temporary fixation are not recommended.

Conclusions. The success of final restorations is mostly conditioned by the proper completion of preceding stages such as fabrication of provisional restorations. The latter protect abutments and periodontal tissues from various harmful factors, restore masticatory function, esthetics, pronunciation defects as well as prevent the displacement of abutments. The choice of provisional restoration fabrication technique depends on the clinical case. Direct technique remains the most preferable due to its advantages in time and cost. Correct choice of provisional restoration fabrication technique and proper sequence of stages enhances the longevity of provisional restorations, contribute to the restoration of the contours of underlying and surrounding tissue, maintain their healthy state as well as provide the patient's contentment and comfort. However, in order to achieve these results and to avoid failures and complications it is necessary to be aware of all advantages, drawbacks and peculiarities of various techniques of provisional restoration fabrication. 


\section{REFERENCES}

1. Glossary of Prosthodontic Terms 8th ed. J Prosthet Dent 2005; 94:10-92.

2. Al Jabbari YS, Al-Rasheed A, Smith JW, Iacopino AM. An indirect technique for assuring simplicity and marginal integrity of provisional restorations during full mouth rehabilitation. Saudi Dental Journal 2013; 25:39-42.

3. Luthardt RG, Stossel M, Hinz M, Vollandt R. Clinical performance and periodontal outcome of temporary crowns and fixed partial dentures: a randomized clinical trial. J Prosthet Dent 2000; 83:32-9.

4. Patras M, Naka O, Doukoudakis S, Pissiotis A. Management of Provisional Restorations' Deficiencies: A Literature Review. Journal of Esthetic and Restorative Dentistry 2011:1-13.

5. Regish KM, Sharma D, Prithviraj DR. Techniques of Fabrication of Provisional Restoration: An Overview. International Journal of Dentistry 2011; 134659.

6. Ayuso-Montero, R., Martinez-Gomis, J., Lujan-Climent, M., Salsench, J. and Peraire, M. (2009) Influence of Matrix Type on Surface Roughness of Three Resins for Provisional Crowns and Fixed Partial Dentures. Journal of Prosthodontics, 18, 141-144.

7. Guler A.U., Kurt S. and Kulunk T. (2005) Effects of Various Finishing Procedures on the Staining of Provisional Restorative Materials. The Journal of Prosthetic Dentistry, 93, 453-458.

8. Christensen GJ. The fastest and best provisional restorations. J Am Dent Assoc 2003; 134:637-9.

9. Sen, D., Göller, G. and Issever, H. (2002) The Effect of Two Polishing Pastes on the Surface Roughness of Bis-Acryl Composite and Methacrylate-Based Resins. The Journal of Prosthetic Dentistry, 88, 527-532.

10. Michalakis K, Pissiotis A, Hirayama H, et al. Comparison of temperature increase in the pulp chamber during the polymerization of materials used for the direct fabrication of provisional restorations. J Prosthet Dent 2006; 96:418-23.

11. Klages U, Bruckner A, Zentner A. Dental esthetics, self-awareness, and oral health-related quality of life in young adults. Eur J Orthod 2004; 26:507-14.

12. Young CW. A simple and predictable direct technique for esthetic provisional veneers. J Calif Dent Assoc. 2004;32(2):178-183

13. Singla M, Padmaja K, Arora J, Shah A. Provisional Restorations in Fixed Prosthodontics: A Review. Int J Dent Med Res 2014;1(4):148-151.

14. H. B. Dumbrigue, "Composite indirect-direct method for fabricating multiple-unit provisional restorations," Journal of Prosthetic Dentistry, vol. 89, no. 1, pp. 86-88, 2003.

15. Burns, D.R, Beck, D.A. and Nelson, S.K. (2003) A Review of Selected Dental Literature on Contemporary Provisional Fixed Prosthodontic Treatment: Report of the Committee on Research in Fixed Prosthodontics of the Academy of Fixed Prosthodontics. The Journal of Prosthetic Dentistry, 90, 474-497.

16. Bennani V. Fabrication of an indirect-direct provisional fixed partial denture. J Prosthet Dent. 2000; 84:364-365

17. Hammond BD, Cooper JR, Lazarchik DA. Predictable repair of provisional restorations. J Esthet Restor Dent 2009; 21:19-25.

18. Buergers, R., Rosentritt, M. and Handel, G. (2007) Bacterial Adhesion of Strept ococcus mutans to Provisional Fixed Prosthodontics Material. The Journal of Prosthetic Dentistry, 98, 461-469.

19. Kuhar, M. and Funduk, N. (2005) Effects of Polishing Techniques on the Surface Roughness of Acrylic Denture Base Resins. The Journal of Prosthetic Dentistry, 93, 76-85.

20. Rüttermann S., Bergmann N., Beikler T., Raab W.H. and Janda R. (2012) Bacterial Viability on SurfaceModified Resin-Based Dental Restorative Materials. Archives of Oral Biology, 57, 1512-1521.

21. Edelhoff D, Spiekermann H, Yildirim M. A review of esthetic pontic design options. Quintessence Int 2002; 33:736-46.

22. Kim TH, Cascione D, Knezevic A. Simulated tissue using a unique pontic design: a clinical report. J Prosthe tDent 2009; 102:205-10.

23. Young HM, Smith CT, Morton D. Comparative in vitro evaluation of two provisional restorative materials. J Prosthet Dent 2001; 85:129-32.

24. Ehrenberg DS, Weiner S. Changes in marginal gap size of provisional resin crowns after occlusal loading and thermal cycling. J Prosthet Dent 2000; 84:139-48.

25. Ehrenberg D, Weiner GI, Weiner S. Long-term effects of storage and thermal cycling on the marginal adaptation of provisional resin crowns: a pilot study. J Prosthet Dent 2006;95:230-6.

26. Chen HL, Lai YL, Chou IC, et al. Shear bond strength of provisional restoration materials repaired with light-cured resins. Oper Den 2008; 33:508-15

27. Burns DR, Beck DA, Nelson SK. A review of selected dental literature on contemporary provisional fixed prosthodontics treatment: report of the committee on research in fixed prosthodontics of the academy of fixed prosthodontics. J Prosthet Dent 2003; 90:474-97.

28. Haselton DR, Diaz-Arnold AM, Vargas MA. Flexural strength of provisional crown and fixed partial denture resins. J Prosthet Dent 2002; 87:225-8.

29. Hagge MS, Lindemuth JS, Jones A. Shear bond strength of bis-acryl composite provisional material repaired with flowable composite. J Esthet Restor Dent 2002; 14:47-52.

30. Appleby DC. Repair of fractured connectors in a provisional fixed partial denture. J Prosthet Dent 2001; 86:449-50. 
31. Guler AU, Kurt S, Kulunk T. Effects of various finishing procedures on the staining of provisional restorative materials. J Prosthet Dent 2005; 93:453-8.

32. Lang R, Rosentritt M, Behr M, Handel G. Fracture resistance of PMMA and resin matrix composite-based interim FPD materials. Int J Prosthodont2003; 16:381-4.

33. Hamza TA, Rosenstiel SF, El-Hosary MM, Ibraheem RM. The effect of fiber reinforcement on the fracture toughness and flexural strength of provisional restorative resins. J Prosthet Dent 2004; 91:258-64.

34. Yilmaz A, Baydaş S. Fracture resistance of various temporary crown materials. J Contemp Dent Pract 2007; 8:44-51.

35. Chen WC, Hung CC, Hyang YC, et al. Fracture load of provisional fixed partial dentures with long-span fiber-reinforced acrylic resin ant thermocycling. J Dent Sci 2009; 4:25-31.

36. Nohrstrom TJ, Vallittu PK, Yli-Urpo A. The effect of placement and quantity of glass fibers on the fracture resistance of interim fixed partial dentures. Int J Prosthodont 2000; 13:72-8.

37. Bohnenkamp DM, Garcia LT. Repair of bis-acryl provisional restorations using flowable composite resin. J Prosthet Dent 2004; 92:500-2.

38. Lee SY, Lai YL, Hsu TS. Influence of polymerization conditions on monomer elution and micro hardness of auto polymerized polymethyl methacrylate resin. Eur J Oral Sci 2002;110:179-83.

39. Lai YL, Chen YT, Lee SY, et al. Cytotoxic effects of dental resin liquids on primary gingival fibroblasts and periodontal ligament cells in vitro. J Oral Rehabil 2004;31:1165-72.

40. Minami H, Suzuki S, Minesaki Y, et al. In vitro evaluation of the influence of repairing condition of denture base resin on the bonding of auto polymerizing resins. J Prosthet Dent 2004; 91:164-70.

41. Nejatidanesh F, Lofti HR, Savabi O. Marginal accuracy of interim restorations fabricated from four interim auto polymerizing resins. J Prosthet Dent 2006; 95:364-7.

42. Wassell RW, St. George G, Ingledew RP, Steele JG. Crowns and other extra coronal restorations: provisional restorations. Br Dent J 2002;192:619-30.

43. Papacchini F, Magni E, Radovic I, et al. Effect of intermediate agents and pre-heating of repairing resin on composite-repair bonds. Oper Dent 2007; 32:363-71.

44. Teixeira EC, Bayne SC, Thompson JY, et al. Shear bond strength of self-etching bonding systems in combination with various composites used for repairing aged composites. J Adhes Dent 2005;7:159-64.

45. Lucena-Martin C, Gonzalez-Lopez S, Navajas-Rodriguez de Mondelo JM. The effect of various surface treatments and bonding agents on the repaired strength of heat-treated composites. J Prosthet Dent 2001; 86:481-8.

46. Balkenhol M, KöhlerH, Orbach K, WöstmannB. Fracture toughness of cross-linked and non-cross-linked temporary crown and fixed partial denture material. Dent Mater 2009; 25:917-28.

47. Bolina J, Lautenschlager EP, Monaghan P. Bond strength of resinous materials to prosthodontic polymeric restoratives. J Dent Res 2005;84:(SpecIssA) abstract No0481.

48. Dumbrigue HB. Composite indirect-direct method for fabricating multiple-unit provisional restorations. J Prosthet Dent 2003; 89:86-8.

49. Patras M., Naka O., Doukoudakis S. and Pissiotis A. (2012), Management of Provisional Restorations' Deficiencies: A Literature Review. Journal of Esthetic and Restorative Dentistry, 24: 26-38. 